\title{
Plasma vitamin D and parathormone are associated with obesity and atherogenic dyslipidemia: a cross-sectional study
}

\author{
Alba Guasch ${ }^{1,2}$, Mònica Bulló 1,3*, Antoni Rabassa², Anna Bonada ${ }^{1,2}$, Daniel Del Castillo ${ }^{4}$, Fàtima Sabench ${ }^{4}$ \\ and Jordi Salas-Salvadó $1,2,3,5^{*}$
}

\begin{abstract}
Background: Low concentrations of plasma vitamin D $(25(\mathrm{OH}) \mathrm{D})$ have been associated with the development of metabolic syndrome (MetS), obesity, diabetes and cardiovascular disease. The objective of this study was to quantify the associations between 25(OH)D and parathormone (PTH) plasma levels and obesity, the presence of MetS, diabetes or atherogenic dyslipidemia (AD) in a large sample of individuals with different degrees of adiposity.

Methods: Retrospective study of all patients who had attended the obesity clinics in a Spanish hospital between 2009 and 2011, and whose concentrations of PTH, 25(OH)D, calcium and alkaline phosphatase had been determined ( $n=316,75.9 \%$ women). Individuals were categorized by degree of adiposity, presence of MetS, and other comorbidities.

Results: PTH increased but 25(OH)D and calcium decreased with increasing adiposity. The prevalence of $25(\mathrm{OH}) \mathrm{D}$ deficiency or insufficiency increased with obesity $\left(<10 \%\right.$ when $\mathrm{BMl}<45 \mathrm{~kg} / \mathrm{m}^{2}$, and $26 \%$ when $\left.>50\right)$. The prevalence of hyperparathyroidism increased from $12 \%$ in non-obese to $47.5 \%$ in morbidly obese individuals with BMl $>50 \mathrm{~kg} / \mathrm{m}^{2}$. Low plasma $25(\mathrm{OH})$ D and high PTH concentrations were associated with an increased risk of MetS and AD. These associations disappeared, except in the case of $A D$ for $25(\mathrm{OH}) \mathrm{D}$ when adjusting for $\mathrm{BMI}$. Regression analysis revealed that $\mathrm{BMI}$ and age or seasonality were independent predictors of PTH and 25(OH)D levels, respectively.

Conclusions: BMI was the variable most strongly associated with plasma 25(OH)D and PTH concentrations in our study. Low 25(OH)D and high PTH concentrations were not independently associated with an increased risk of MetS, or diabetes. Our data support a possible contribution of plasma 25(OH)D to the pathogenesis of hypertriglyceridemia and AD through inflammation.
\end{abstract}

Keywords: Obesity, Metabolic syndrome, Vitamin D, Parathormone

\section{Background}

Obesity has become a serious health problem in most developed countries. The World Health Organization estimated that in 2008 the global prevalence of overweight and obesity was around 1.5 billion and 500 million adults, respectively. Obesity is also well known to be associated with an increase in the prevalence of type 2

\footnotetext{
*Correspondence: monica.bullo@urv.cat; jordi.salas@urv.cat

'Human Nutrition Unit, Faculty of Medicine and Health Sciences, IISPV,

Universitat Rovira i Virgili, Reus, Spain

${ }^{3} \mathrm{ClBER}$ obn Physiopathology of Obesity and Nutrition, Institute of Health

Carlos III, Madrid, Spain

Full list of author information is available at the end of the article
}

diabetes, cardiovascular diseases, certain types of cancer and total mortality [1].

Vitamin D deficiency is also an important worldwide public health problem [2]. Although the most-studied and best-known function of vitamin $\mathrm{D}$, together with parathyroid hormone (PTH), is related to bone metabolism [3], many studies show evidence of the relationship between obesity and low levels of 25(OH)D (the best indicator of clinical levels of vitamin D) $[4,5]$. Factors known to influence 25(OH)D concentrations include race, vitamin $\mathrm{D}$ intake, sun exposure, adiposity, age and physical activity [3]. In obese people, low levels of 25 $(\mathrm{OH}) \mathrm{D}$ can be attributed mainly to: a) the lower 
bioavailability of the vitamin, due to its sequestration by adipose tissue [6]; b) the dilution of ingested or cutaneously synthesized vitamin $D$ in the enlarged fat mass [7]; c) low sun exposure, due to mobility limitations or the low sun exposure of large areas of the body [8]; or d) a low intake of calcium and vitamin D. The frequently observed increases in PTH serum concentrations in obese individuals [9] could be explained by a compensatory mechanism in response to low circulating levels of 25(OH)D.

More recently, vitamin D deficiency has been related to the pathogenesis of such comorbidities as insulin resistance [10], type 2 diabetes [11], hypertension [12], dyslipidemia [13], and cardiovascular diseases [14]. A controversial association has been reported between low levels of plasma 25(OH)D and/or elevated PTH and the presence of metabolic syndrome (MetS) and its individual components,. Some cross-sectional studies note that decreased levels of $25(\mathrm{OH}) \mathrm{D}$ are associated to a higher risk of MetS after adjusting for various potential confounding factors $[15,16]$. A recent systematic review and meta-analysis showed that the prevalence of MetS was reduced by approximately $50 \%$ if individuals had high 25 $(\mathrm{OH}) \mathrm{D}$ concentrations [17]. Other studies showed that high concentrations of $\mathrm{PTH}$, but not $25(\mathrm{OH}) \mathrm{D}$, were associated with the presence of the syndrome $[18,19]$. However, no association has been reported between PTH or 25(OH)D and MetS in overweight or obese individuals [20].

In this context, the aim of our study was to evaluate the associations between $25(\mathrm{OH}) \mathrm{D}$ or PTH concentrations and the risk of obesity, and MetS and its individual components in a large sample of individuals with a wide range of adiposity.

\section{Methods}

\section{Study population}

The medical records of all patients attending the obesity outpatient clinics of the Hospital Sant Joan de Reus between September 2009 and December 2011 were retrospectively reviewed. Caucasian individuals who had had at least one blood test that simultaneously measured plasma concentrations of PTH, $25(\mathrm{OH}) \mathrm{D}$, calcium, phosphate and alkaline phosphatase were included in the present study.

Exclusion criteria were bariatric surgery before the blood test, any severe chronic illness, drug or alcohol addiction, endocrine disease (except type 2 diabetes mellitus or treated hyperthyroidism), cancer in the previous 5 years, active concomitant inflammatory disease, advanced liver disease, chronic renal failure and continued drug treatment with derivatives of vitamin $\mathrm{D}$ and calcium. Subjects were also excluded when primary hyperparathyroidism was suspected, when plasma concentrations of PTH were high $(>6.85 \mathrm{pmol} / \mathrm{L})$ and hypercalcemia (> $2.5 \mathrm{mmol} / \mathrm{L}$ ) and/or hypophosphatemia $(<0.8 \mathrm{mmol} / \mathrm{L})$ were present simultaneously. The study was conducted in agreement with the 1990 Declaration of Helsinki and subsequent amendments. The Sant Joan Hospital Ethical Committee from Reus (Spain) approved the study protocol.

\section{Design}

A cross-sectional study was conducted on a population of individuals with a wide range of adiposity. From the medical records of the individuals selected we collected the following data: age, gender, current smoking (absent/ present), presence of comorbidities associated with obesity (type 2 diabetes, hypertension, dyslipidemia), medication use (fibrates, statins and other lipid lowering agents, oral antidiabetic agents, insulin and antihypertensive drugs) and seasonality of blood sampling (winter: December-February, spring: March-May, summer: JuneAugust, autumn: September-November). Weight and height were measured with individuals wearing light clothing and without shoes using calibrated scales and a wall-mounted stadiometer (scale capacity of $220 \mathrm{~kg}$ and a precision of $50 \mathrm{~g}$, and a measuring range between $60-200 \mathrm{~cm}$ with an accuracy of $1 \mathrm{~mm}$ ). BMI was calculated by dividing the weight (in $\mathrm{kg}$ ) by the square of the height (in meters). Blood pressure was measured with a mercury sphygmomanometer adapted to the subject's BMI, and after the subject had been seated for at least five minutes.

Individuals were classified into different categories of adiposity using the BMI-based index of the Spanish Society for the Study of Obesity (SEEDO) [21]. The MetS was defined according to the updated NCEP-ATP III criteria [22], which require three or more of the following conditions to be met: abdominal obesity (waist circumference $>102 \mathrm{~cm}$ in men and $>88 \mathrm{~cm}$ in women), hypertriglyceridemia (triglyceride level $150 \mathrm{mg} / \mathrm{dL}$ or 1.7 $\mathrm{mmol} / \mathrm{L}$ or taking triglyceride-lowering medication), low HDL cholesterol $(<40 \mathrm{mg} / \mathrm{dL}$ or $1.0 \mathrm{mmol} / \mathrm{L}$ in men and $<50 \mathrm{mg} / \mathrm{dL}$ or $1.3 \mathrm{mmol} / \mathrm{L}$ in women), elevated fasting blood glucose $(100 \mathrm{mg} / \mathrm{dL}$ or $5.6 \mathrm{mmol} / \mathrm{L}$ or taking antidiabetic medication), and elevated blood pressure (systolic $130 \mathrm{~mm} \mathrm{Hg}$, diastolic $85 \mathrm{~mm} \mathrm{Hg}$, or taking antihypertensive medication). Participants who were being treated with antidiabetic, antihypertensive or triglyceride-lowering medication were considered to be diabetic, hypertensive, or hypertriglyceridemic, respectively. Atherogenic dyslipidemia was defined when high plasma triglycerides and low HDL-concentrations were concomitantly present. We considered that those patients for whom we had no waist circumference measurement complied with the criterion of abdominal obesity when their BMI was higher than $35 \mathrm{~kg} / \mathrm{m}^{2}$. 


\section{Biochemical determinations}

Blood samples were obtained after an overnight fast. Plasma concentrations of glucose, calcium, phosphate, alkaline phosphatase, total cholesterol, HDL cholesterol, LDL cholesterol, triglycerides, creatinine, serum albumin, erythrocyte sedimentation rate (ESR) and leukocyte count were determined following routine biochemical laboratory protocols. The corrected calcium level based on the serum albumin concentrations was calculated as: Corrected calcium $(\mathrm{mmol} / \mathrm{L})=$ measured total $\mathrm{Ca}$ $(\mathrm{mmol} / \mathrm{L})+0.02(40-$ serum albumin $[\mathrm{g} / \mathrm{L}])$, where 40 represents the average albumin level in $\mathrm{g} / \mathrm{L}$. The ultrasensitive C Reactive Protein (uCRP) was determined by immunoturbidimetry. Serum 25(OH)D was determined by electrochemiluminiscence immunoassay using a Cobas autoanalyzer (Roche Diagnostics, West Sussex, UK; intra- and inter-assay CVs $<8 \%$ and $<10 \%$, respectively). Intact PTH were determined by chemiluminescent enzyme-labeled immunometric assay using an IMMULITE 2000 Systems analyzer (Siemens, Gwynedd, UK; intra- and inter-assay CVs $<5 \%$ and $<8 \%$, respectively).

The normal, insufficient and deficient vitamin D statuses were defined as serum concentrations of $25(\mathrm{OH}) \mathrm{D}$ $>75.00 \mathrm{nmol} / \mathrm{L}$, between 25.00 and $75.00 \mathrm{nmol} / \mathrm{L}$, and $<25.00 \mathrm{nmol} / \mathrm{L}$, respectively [23]. Secondary hyperparathyroidism was diagnosed in individuals with serum concentrations of $\mathrm{PTH}>6.85 \mathrm{pmol} / \mathrm{L}$.

\section{Statistical analysis}

Data are given as means (95\% confidence interval, 95\% CI) for continuous variables or percentages for categorical variables, unless otherwise stated. Differences between groups were tested using one way analysis of variance (ANOVA) for continuous data and the chi-square test $\left(x^{2}\right)$ for categorical data. The studied population was divided into six BMI ranges $(<30,30-35,35-40,40-45$, $45-50$ and $>50 \mathrm{Kg} / \mathrm{m}^{2}$ ). To evaluate predictors of $25(\mathrm{OH})$ $\mathrm{D}$ and $\mathrm{PTH}$, a regression analysis was performed with age, gender, BMI, plasma HDL and LDL cholesterol, triglycerides, glucose, the season when the blood sample was obtained [categorized as summer (March to August) or winter (September to February)], and presence of hypertension as covariates (Model 1). In a separate analysis (Model 2), uCRP and leucocytes were added to Model 1 as covariates.

Multiple logistic regressions with predefined explanatory variables were used to assess odds for MetS, components of the MetS and atherogenic dyslipidemia. Four separate multiple logistic regression models were fitted. In the first model, serum PTH, 25(OH)D and calcium were entered into a multiple logistic regression analysis with MetS (yes/no), presence of MetS components (yes/ no) and atherogenic dyslipidemia (yes/no) as dependent variables. In the second model, age, gender, season of blood sampling and current smoking were added to model 1 as confounding variables. In the third model (model 3), BMI was also included. In the fourth model, uCRP and leucocytes were included. A statistical significance level of 5\% was chosen. The analyses were implemented using the SPSS 19.0 statistical package.

\section{Results}

The general characteristics of the studied population are shown in Table 1. A total of 316 patients met the inclusion criteria and were included in the analyses. Of these $75.9 \%(\mathrm{n}=240)$ were women. The prevalence of MetS was $62.0 \%$, with no significant differences between the sexes. High blood pressure was the most common feature of MetS observed in both sexes. Blood pressure and plasma concentrations of glucose, triglycerides, albumin and creatinine were significantly higher, and plasma HDL and LDL cholesterol, and ESR were lower in men than in women. Women showed lower plasma concentrations of $25(\mathrm{OH}) \mathrm{D}$ and calcium than males.

Table 2 describes the characteristics of the patients in the various BMI ranges. A higher proportion of women were observed among those with a BMI higher than 35 $\mathrm{kg} / \mathrm{m}^{2}$. The prevalence of MetS and its features (abdominal obesity, elevated blood pressure, hyperglycemia, and low HDL-c) increased when the BMI range was increased. Although there were no significant differences in blood pressure, LDL cholesterol and plasma triglycerides across BMI ranges, plasma glucose, ultrasensitive uCRP, ESR and leukocyte counts increased and HDL cholesterol and albumin decreased when the BMI range increased.

Significant mean differences in plasma calcium $(\mathrm{P}<0.046), 25(\mathrm{OH}) \mathrm{D}(\mathrm{P}<0.001)$ and $\mathrm{PTH}(\mathrm{P}<0.001)$ concentrations were observed across the individuals categorized by BMI. Individuals with lower BMI had higher plasma concentrations of $25(\mathrm{OH}) \mathrm{D}$ and calcium than individuals with higher BMI (Table 2). The concentrations of PTH increased as BMI ranges increased. No mean differences were found in plasma phosphate and alkaline phosphatase concentrations between BMI groups.

Table 3 shows the vitamin D status and the prevalence of secondary hyperparathyroidism for the BMI categories. The prevalence of vitamin D deficiency and insufficiency increased with obesity (by $<10 \%$ in individuals with BMI $<45 \mathrm{~kg} / \mathrm{m}^{2}$, and $26 \%$ in those $>50 \mathrm{~kg} / \mathrm{m}^{2}$ ). When vitamin $\mathrm{D}$ deficiency and insufficiency were merged, only $38 \%$ of individuals with a BMI lower than $30 \mathrm{~kg} / \mathrm{m}^{2}$ had vitamin D insufficiency or deficiency, compared to $88-95 \%$ of those with a BMI higher than 35 
Table 1 Characteristics of the study population

\begin{tabular}{|c|c|c|c|c|}
\hline Variables & Total $(n=316)$ & Men $(n=76)$ & Women $(n=240)$ & $\mathrm{P}^{*}$ \\
\hline Age (years) & $46.85(45.44,48.26)$ & $49.28(46.31,52.24)$ & $46.08(44.49,47.68)$ & 0.056 \\
\hline Weight (Kg) & $105.29(102.30,108.28)$ & $111.06(103.77,118.36)$ & $103.46(100.28,106.65)$ & 0.032 \\
\hline $\mathrm{BMI}\left(\mathrm{Kg} / \mathrm{m}^{2}\right)$ & $40.02(38.94,41.09)$ & $37.67(35.29,40.05)$ & $40.76(39.56,41.96)$ & 0.016 \\
\hline Presence of metabolic syndrome & $62.0 \%$ & $61.8 \%$ & $62.1 \%$ & 1.000 \\
\hline Abdominal obesity & $69.9 \%$ & $50.0 \%$ & $75.8 \%$ & $<0.001$ \\
\hline Elevated blood pressure & $83.4 \%$ & $91.4 \%$ & $80.4 \%$ & 0.039 \\
\hline Elevated fasting glucose & $55.6 \%$ & $61.3 \%$ & $53.8 \%$ & 0.287 \\
\hline Reduced HDL cholesterol & $61.8 \%$ & $54.2 \%$ & $64.1 \%$ & 0.165 \\
\hline Elevated fasting triglycerides & $30.0 \%$ & $45.1 \%$ & $25.4 \%$ & 0.003 \\
\hline Current smoking & $20.6 \%$ & $21.1 \%$ & $20.4 \%$ & 0.620 \\
\hline Seasonality (spring-summer) & $39.2 \%$ & $40.8 \%$ & $38.8 \%$ & 0.788 \\
\hline \multicolumn{5}{|l|}{ Drugs } \\
\hline Fibrates & $2.3 \%$ & $4.3 \%$ & $1.7 \%$ & 0.195 \\
\hline Oral antidiabetic & $18.0 \%$ & $27.6 \%$ & $15.0 \%$ & 0.016 \\
\hline Insulin & $2.8 \%$ & $5.3 \%$ & $2.1 \%$ & 0.226 \\
\hline Antihypertensive & $34.2 \%$ & $48.7 \%$ & $29.6 \%$ & 0.003 \\
\hline Systolic blood pressure $(\mathrm{mmHg})$ & $139.99(137.35,142.63)$ & $146.39(140.83,151.95)$ & $137.61(134.68,140.54)$ & 0.003 \\
\hline Diastolic blood pressure (mmHg) & $84.01(82.50,85.52)$ & $87.68(84.88,90.47)$ & $82.65(80.89,84.41)$ & 0.003 \\
\hline Glucose (mmol/L) & $6.05(5.83,6.27)$ & $6.57(5.99,7.15)$ & $5.88(5.66,6.11)$ & 0.009 \\
\hline HDL cholesterol (mmol/L) & $1.22(1.17,1.26)$ & $1.06(0.97,1.15)$ & $1.27(1.21,1.32)$ & $<0.001$ \\
\hline LDL cholesterol (mmol/L) & $3.22(3.11,3.32)$ & $2.91(2.68,3.14)$ & $3.31(3.19,3.42)$ & 0.002 \\
\hline Triglycerides (mmol/L) & $1.48(1.37,1.58)$ & $1.74(1.45,2.02)$ & $1.39(1.29,1.49)$ & 0.005 \\
\hline ALAT ( $\mu$ Kat/L) & $0.36(0.34,0.38)$ & $0.41(0.37,0.45)$ & $0.35(0.33,0.37)$ & 0.014 \\
\hline GT ( $\mu$ Kat/L) & $0.44(0.40,0.49)$ & $0.59(0.45,0.73)$ & $0.41(0.36,0.45)$ & 0.001 \\
\hline Leucocyte count (x10E9/L) & $7.69(7.43,7.95)$ & $7.43(6.75,8.10)$ & $7.76(7.47,8.04)$ & 0.324 \\
\hline Albumin (g/L) & $40.25(39.70,40.81)$ & $42.04(40.66,43.41)$ & $39.80(39.21,40.39)$ & 0.001 \\
\hline CRP ultrasensitive (mg/dL) & $9.41(8.30,10.52)$ & $8.53(5.37,11.69)$ & $9.62(8.46,10.79)$ & 0.444 \\
\hline $\operatorname{ESR}(\mathrm{mm} / \mathrm{h})$ & $22.67(20.61,24.74)$ & $13.10(10.04,16.17)$ & $25.03(22.68,27.38)$ & $<0.001$ \\
\hline Calcium (mmol/L) & $2.34(2.33,2.35)$ & $2.36(2.34,2.38)$ & $2.33(2.32,2.34)$ & 0.020 \\
\hline Phosphate (mmol/L) & $1.16(1.13,1.18)$ & $1.13(1.08,1.18)$ & $1.16(1.14,1.19)$ & 0.294 \\
\hline Alkaline phosphatase (uKat/L) & $1.23(1.17,1.29)$ & $1.21(1.06,1.36)$ & $1.24(1.17,1.31)$ & 0.656 \\
\hline Creatinine (mol/L) & $63.33(61.55,65.12)$ & $80.00(75.81,84.20)$ & $59.19(57.63,60.74)$ & $<0.001$ \\
\hline eGFR $\left(\mathrm{ml} / \mathrm{min} / 1.73 \mathrm{~m}^{2}\right)$ & $103.08(101.16,105.20)$ & $98.86(93.89,103.83)$ & $104.25(102.06,106.44)$ & 0.035 \\
\hline PTH (pmol/L) & $5.83(5.53,6.14)$ & $5.42(4.84,5.99)$ & $5.97(5.61,6.32)$ & 0.129 \\
\hline $25(\mathrm{OH}) \mathrm{D}(\mathrm{nmol} / \mathrm{L})$ & $56.28(51.58,60.98)$ & $65.01(53.18,76.83)$ & $53.55(48.60,58.49)$ & 0.041 \\
\hline
\end{tabular}

Data are means $(95 \% \mathrm{Cl})$ for continuous variables and \% for categorical variables.

*P value comparing males and females: One-way analysis of variance (ANOVA) for continuous variables, and chi-square test $\left(X^{2}\right)$ for categorical variables.

Abbreviations: BMI: Body Mass Index; HDL: High Density Lipoprotein; LDL: Low Density Lipoprotein; ALAT: Alanine transaminase; GT: Glutamyl transaminase; CRP: C Reactive Protein; ESR: Erythrocyte Sedimentation Rate; eGFR: Estimated Glomerular Filtration Rate; PTH Parathormone; 25(OH)D; 25 Hydroxy-cholecalcipherol.

$\mathrm{kg} / \mathrm{m}^{2}$. The prevalence of hyperparathyroidism increased from $12 \%$ in non-obese subjects to $47.5 \%$ in those with a BMI $>50 \mathrm{~kg} / \mathrm{m}^{2}$.

In a multivariate analysis (Table 4), BMI, age and HDL-cholesterol were independent predictors of serum PTH levels, and together they explain $21 \%$ of the variance in PTH concentrations. For plasma 25(OH)D concentrations, BMI and blood sample seasonality were the independent predictors, explaining $17 \%$ of their variability. When uCRP and leukocyte counts were added to the model, BMI and uCRP levels explained $12 \%$ of the variability of $25(\mathrm{OH}) \mathrm{D}$ concentrations.

In a multiple logistic regression (Table 5), PTH levels were associated with significantly higher odds of MetS in 
Table 2 Characteristics of patients according to body mass index $\left(\mathrm{kg} / \mathrm{m}^{2}\right)$ ranges

\begin{tabular}{|c|c|c|c|c|c|c|c|}
\hline & & & & BMI $\left(\mathrm{Kg} / \mathrm{m}^{2}\right)$ & & & \\
\hline & $<30(n=50)$ & $30-35(n=45)$ & $35-40(n=45)$ & $40-45(n=81)$ & $45-50(n=53)$ & $>50(n=42)$ & $\mathrm{P}^{*}$ \\
\hline Age (years) & $45.2(40.2,50.2)$ & $53.0(49.3,56.7)^{\#}$ & $44.8(41.4,48.3)$ & $45.99(43.5,48.5)$ & $46.1(42.9,49.2)$ & $47.3(44.3,50.4)$ & 0.022 \\
\hline Women & $60.8 \%$ & $60.0 \%$ & $80.0 \%$ & $82.7 \%$ & $84.9 \%$ & $83.3 \%$ & 0.002 \\
\hline Weight (Kg) & $66.6(63.3,69.9)$ & $87.2(84.4,90.1) \#$ & $99.5 \underset{\#{ }^{\prime}}{(95.9,103.1)}$ & $112.2 \underset{\# \#}{(109.4,114.9)}$ & $122.2 \underset{\# \#}{(118.5,125.8)}$ & $143.1 \underset{\# \#}{(136.2,145.00)}$ & $<0.001$ \\
\hline BMI $\left(\mathrm{Kg} / \mathrm{m}^{2}\right)$ & $24.9(23.9,26.00)$ & $32.4(31.9,32.8){ }^{\# \#}$ & $37.3(36.8,37.7){ }^{\# \#}$ & $42.7(42.4,43.0)^{\# \#}$ & $47.2(46.8,47.6){ }^{\# \#}$ & $55.2(53.5,56.8){ }^{\# \#}$ & $<0.001$ \\
\hline Presence of MetS & $19.6 \%$ & $35.6 \%$ & $57.8 \%$ & $79.0 \%$ & $79.2 \%$ & $92.9 \%$ & $<0.001$ \\
\hline $\begin{array}{l}\text { Abdominal } \\
\text { obesity }\end{array}$ & $0.0 \%$ & $4.4 \%$ & $100.0 \%$ & $100.0 \%$ & $100.0 \%$ & $100.0 \%$ & $<0.001$ \\
\hline $\begin{array}{l}\text { Elevated blood } \\
\text { pressure }\end{array}$ & $59.2 \%$ & $83.3 \%$ & $78.8 \%$ & $93.5 \%$ & $92.7 \%$ & $93.9 \%$ & $<0.001$ \\
\hline $\begin{array}{l}\text { Elevated fasting } \\
\text { glucose }\end{array}$ & $31.4 \%$ & $55.8 \%$ & $45.5 \%$ & $60.0 \%$ & $63.5 \%$ & $78.6 \%$ & $<0.001$ \\
\hline $\begin{array}{l}\text { Reduced HDL } \\
\text { cholesterol }\end{array}$ & $14.0 \%$ & $50.0 \%$ & $60.5 \%$ & $76.6 \%$ & $80.4 \%$ & $83.3 \%$ & $<0.001$ \\
\hline $\begin{array}{l}\text { Elevated fasting } \\
\text { triglycerides }\end{array}$ & $25.5 \%$ & $39.0 \%$ & $27.9 \%$ & $25.0 \%$ & $35.5 \%$ & $33.3 \%$ & 0.556 \\
\hline $\begin{array}{l}\text { Systolic blood } \\
\text { pressure (mmHg) }\end{array}$ & $\begin{array}{l}136.4(128.5 \\
144.3)\end{array}$ & $\begin{array}{l}141.4(136.00 \\
146.8)\end{array}$ & $\begin{array}{l}133.8(127.4 \\
140.2)\end{array}$ & $142.4(137.5,147.4)$ & $141.5(136.1,147.0)$ & $144.2(136.2,152.2)$ & 0.244 \\
\hline $\begin{array}{l}\text { Diastolic blood } \\
\text { pressure (mmHg) }\end{array}$ & $80.1(76.6,83.7)$ & $85.6(81.8,89.3)$ & $82.4(77.9,87.00)$ & $85.3(82.5,88.2)$ & $84.4(80.2,88.7)$ & $87.0(82.8,91.2)$ & 0.087 \\
\hline Glucose (mmol/L) & $5.34(4.88,5.79)$ & $6.05(5.59,6.51)$ & $5.80(5.35,6.26)$ & $6.08(5.63,6.53)$ & $6.13(5.59,6.68)$ & $7.09(6.21,7.97){ }^{\# \#}$ & 0.002 \\
\hline $\begin{array}{l}\text { HDL cholesterol } \\
\text { (mmol/L) }\end{array}$ & $1.60(1.47,1.73)$ & $1.21(1.11,1.32)^{\# \#}$ & $1.24(1.11,1.36)^{\# \#}$ & $1.12(1.05,1.20)^{\# \#}$ & $1.07(0.97,1.18)^{\# \#}$ & $1.10(1.02,1.17){ }^{\# \#}$ & $<0.001$ \\
\hline $\begin{array}{l}\text { LDL cholesterol } \\
(\mathrm{mmol} / \mathrm{L})\end{array}$ & $2.87(2.58,3.16)$ & $3.27(2.95,3.59)$ & $3.45(3.18,3.72)$ & $3.19(3.01,3.36)$ & $3.32(3.05,3.59)$ & $3.23(2.93,3.53)$ & 0.070 \\
\hline $\begin{array}{l}\text { Triglycerides } \\
\text { (mmol/L) }\end{array}$ & $1.26(0.99,1.54)$ & $1.56(1.25,1.87)$ & $1.54(1.13,1.95)$ & $1.43(1.28,1.58)$ & $1.61(1.40,1.82)$ & $1.52(1.26,1.77)$ & 0.464 \\
\hline ALAT ( $\mu$ Kat/L) & $0.35(0.29,0.41)$ & $0.32(0.30,0.35)$ & $0.35(0.32,0.38)$ & $0.36(0.33,0.39)$ & $0.38(0.31,0.45)$ & $0.40(0.34,0.45)$ & 0.430 \\
\hline GT ( $\mu$ Kat/L) & $0.27(0.21,0.32)$ & $0.45(0.27,0.63)$ & $0.47(0.35,0.59)$ & $0.48(0.38,0.59)$ & $0.44(0.37,0.50)$ & $0.48(0.39,0.57)$ & 0.131 \\
\hline $\begin{array}{l}\text { Leucocyte count } \\
\text { (x10E9/L) }\end{array}$ & $6.08(5.47,6.70)$ & $6.17(5.65,6.70)$ & $7.93(7.12,8.74) \#$ & $7.99(7.53,8.44){ }^{\# \#}$ & $8.26(7.66,8.86)^{\# \#}$ & $8.63(8.01,9.25))^{\# \#}$ & $<0.001$ \\
\hline Albumin $(g / L)$ & $47.0(45.5,48.5)$ & $41.9(40.4,43.3) \#$ & $40.6(39.4,41.8){ }^{\# \#}$ & $38.8(38.00,39.6){ }^{\# \#}$ & $38.4(37.3,39.5){ }^{\# \#}$ & $38.8(37.5,40.1){ }^{\# \#}$ & $<0.001$ \\
\hline $\begin{array}{l}\text { CRP ultrasensitive } \\
\text { (mg/dL) }\end{array}$ & $2.67(1.90,3.45)$ & $4.17(2.71,5.63)$ & $7.75(5.81,9.68)$ & $9.35(7.27,11.44)$ \# & $11.16(8.77,13.54){ }^{\#}$ & $16.80(13.07,20.53) \#$ & $<0.001$ \\
\hline ESR $(\mathrm{mm} / \mathrm{h})$ & $\begin{array}{c}14.50(10.15 \\
18.85)\end{array}$ & $18.74(14.56,22.92)$ & $\begin{array}{l}18.38(13.70 \\
23.05)\end{array}$ & $21.03(17.93,24.12)$ & $26.95(21.94,31.96)$ \# & $34.84(27.16,42.52){ }^{\# \#}$ & $<0.001$ \\
\hline 25(OH)D (nmol/L) & $95.8(76.0,115.5)$ & $59.1(48.5,69.7) \# \#$ & $48.2(42.3,54.0){ }^{\# \#}$ & $49.8(42.6,57.1){ }^{\# \#}$ & $46.6(40.0,53.2){ }^{\# \#}$ & $37.6(30.3,44.8){ }^{\# \#}$ & $<0.001$ \\
\hline PTH (pmol/L) & $4.27(3.61,4.93)$ & $5.11(4.50,5.72)$ & $5.84(5.07,6.61)$ & $6.05(5.50,6.60)^{\#}$ & $6.39(5.68,7.10))^{\#}$ & $7.41(6.31,8.52){ }^{\# \#}$ & $<0.001$ \\
\hline Calcium (mmol/L) & $2.38(2.34,2.41)$ & $2.32(2.29,2.35)$ & $2.32(2.30,2.35)$ & $2.33(2.31,2.35)$ & $2.33(2.31,2.36)$ & $2.34(2.31,2.36)$ & 0.046 \\
\hline $\begin{array}{l}\text { Phosphate } \\
\text { (mmol/L) }\end{array}$ & $1.20(1.12,1.27)$ & $1.11(1.03,1.19)$ & $1.19(1.13,1.26)$ & $1.14(1.10,1.18)$ & $1.17(1.11,1.22)$ & $1.16(1.10,1.21)$ & 0.374 \\
\hline $\begin{array}{l}\text { Alkaline phosphatase } \\
\text { (uKat/L) }\end{array}$ & $1.14(1.04,1.25)$ & $1.13(1.02,1.24)$ & $1.30(1.15,1.46)$ & $1.23(1.10,1.35)$ & $1.21(1.03,1.38)$ & $1.42(1.19,1.64)$ & 0.132 \\
\hline $\begin{array}{l}\text { eGFR } \\
\left(\mathrm{ml} / \mathrm{min} / 1.73 \mathrm{~m}^{2}\right)\end{array}$ & $105.1(99.3,110.8)$ & $99.1(91.6,106.6)$ & $101.9(97.1,106.6)$ & $105.0(101.7,108.4)$ & $103.2(98.0,108.5)$ & $102.4(96.7,108.1)$ & 0.627 \\
\hline
\end{tabular}

Data are means $(\mathrm{Cl})$ for continuous variables and \% for categorical variables.

* $\mathrm{P}$ value by one-way analysis of variance (ANOVA) for continuous variables, and chi-square test $\left(\mathrm{X}^{2}\right)$ for categorical variables.

\# Statistical significance compared to the first BMl group $\left(B M l<30 \mathrm{Kg} / \mathrm{m}^{2}\right)$ : " $\mathrm{P}<0.05$ and \#\# $\mathrm{P}<0.001$ by ANOVA, Bonferroni posthoc test.

Abbreviations: BMI: Body Mass Index; MetS: Metabolic Syndrome; HDL: High Density Lipoprotein; LDL: Low Density Lipoprotein; ALAT: Alanine transaminase;

GT: Glutamyl transaminase; UCRP: ultrasensitive C Reactive Protein; ESR: Erythrocyte Sedimentation Rate; PTH Parathormone; 25(OH)D; 25.Hydroxy-cholecalcipherol; eGFR: Estimated Glomerular Filtration Rate. 
Table 3 Vitamin D status and presence of hyperparathyroidism according to body mass index $\left(\mathrm{kg} / \mathrm{m}^{2}\right) \mathrm{ranges}$

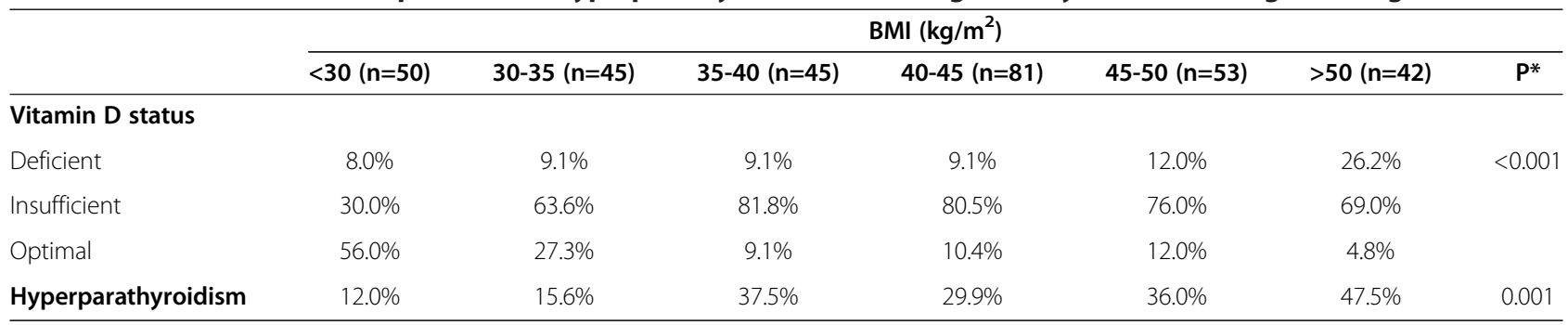

* $P$ value from the Chi-square test $\left(x^{2}\right)$.

Abbreviations: $B M I$ : Body Mass Index.

Vitamin D status by 25(OH)D concentration: deficient $(<25 \mathrm{nmol} / \mathrm{L})$, insufficient $(25-75 \mathrm{nmol} / \mathrm{L})$ and optimal ( $>75 \mathrm{nmol} / \mathrm{L})$.

Hyperparathyroidism: parathyroid hormone concentration $>6.85 \mathrm{pmol} / \mathrm{L}$.

model 1, but not after adjustment for other confounders (models 2, 3 and 4). PTH was also positively associated with elevated blood pressure and reduced HDL cholesterol components of the MetS (models 1 and 2). However, these associations disappeared when adjusted for BMI. PTH was associated with higher odds of atherogenic dyslipidemia in both model 1 and model 2, but not after adjustment for BMI (model 3). High 25(OH)D levels were significantly associated with lower odds of MetS, and reduced HDL cholesterol and diabetes/hyperglycemia components of the MetS in models 1 or 2, but not after the final adjustment for BMI (models 3 and 4). High 25(OH)D levels were significantly associated with lower odds of elevated triglyceride components of the

Table 4 Predictors of PTH and 25(OH)D by multiple regression analysis

\begin{tabular}{|c|c|c|c|c|c|c|c|c|c|}
\hline Model 1 & $\beta$ & $95 \% \mathrm{Cl}$ & $\mathrm{P}^{*}$ & $\mathrm{R}^{2}$ & Model 2 & $\beta$ & $95 \% \mathrm{Cl}$ & $\mathbf{P}^{*}$ & $\mathrm{R}^{2}$ \\
\hline PTH (pmol/L) $(n=229)$ & & & & & PTH (pmol/L) $(n=180)$ & & & & \\
\hline $\mathrm{BMI}\left(\mathrm{kg} / \mathrm{m}^{2}\right)$ & 0.331 & $0.045,0.119$ & $<0.001$ & & $\mathrm{BMI}\left(\mathrm{kg} / \mathrm{m}^{2}\right)$ & 0.291 & $0.032-0.129$ & 0.001 & \\
\hline Age (years) & 0.263 & $0.025,0.079$ & $<0.001$ & & Age (years) & 0.187 & $0.007,0.070$ & 0.018 & \\
\hline Gender & 0.050 & $-0.471,1.097$ & 0.433 & & Gender & 0.062 & $-0.609,1.438$ & 0.425 & \\
\hline $\mathrm{c}-\mathrm{HDL}(\mathrm{mmol} / \mathrm{L})$ & -0.162 & $-1.959,-0.129$ & 0.026 & & $\mathrm{c}-\mathrm{HDL}(\mathrm{mmol} / \mathrm{L})$ & -0.171 & $-2.403,-0.029$ & 0.045 & \\
\hline $\mathrm{c}-\mathrm{LDL}(\mathrm{mmol} / \mathrm{L})$ & 0.077 & $-0.140,0.584$ & 0.228 & 0.211 & $\mathrm{c}-\mathrm{LDL}(\mathrm{mmol} / \mathrm{L})$ & 0.067 & $-0.235,0.625$ & 0.373 & \\
\hline $\mathrm{TG}(\mathrm{mmol} / \mathrm{L})$ & -0.093 & $-0.851,0.141$ & 0.159 & & $\mathrm{TG}(\mathrm{mmol} / \mathrm{L})$ & -0.134 & $-1.114,0.103$ & 0.103 & 0.146 \\
\hline Glucose (mmol/L) & -0.092 & $-0.301,0.058$ & 0.183 & & Glucose (mmol/L) & -0.077 & $-0.308,0.110$ & 0.350 & \\
\hline Seasonality & -0.138 & $-1.415,-0.102$ & 0.024 & & Seasonality & -0.101 & $-1.316,0.221$ & 0.162 & \\
\hline \multirow[t]{3}{*}{ Hypertension } & 0.031 & $-0.735,1.168$ & 0.654 & & Hypertension & 0.044 & $-0.803,1.420$ & 0.584 & \\
\hline & & & & & $\mathrm{CRP}(\mathrm{mg} / \mathrm{dL})$ & -0.034 & $-0.060,0.039$ & 0.672 & \\
\hline & & & & & Leucocyte count (x10E9/L) & 0.027 & $-0.153,0.218$ & 0.731 & \\
\hline $25(\mathrm{OH}) \mathrm{D}(\mathrm{nmol} / \mathrm{L})(\mathrm{n}=234)$ & & & & & $25(\mathrm{OH}) \mathrm{D}(\mathrm{nmol} / \mathrm{L})(\mathrm{n}=186)$ & & & & \\
\hline $\mathrm{BMI}\left(\mathrm{kg} / \mathrm{m}^{2}\right)$ & -0.324 & $-2.103,-0.801$ & $<0.001$ & & $\mathrm{BMI}\left(\mathrm{kg} / \mathrm{m}^{2}\right)$ & -0.238 & $-1.357,-0.212$ & 0.008 & \\
\hline Seasonality & -0.151 & $-25.459,-2.834$ & 0.014 & & $\mathrm{CRP}(\mathrm{mg} / \mathrm{dL})$ & -0.195 & $-1.289,-0.133$ & 0.016 & \\
\hline Age (years) & 0.032 & $-0.341,0.560$ & 0.633 & & Age (years) & 0.013 & $-0.334,0.395$ & 0.869 & \\
\hline Gender & -0.112 & $-25.299,1.781$ & 0.088 & & Gender & -0.039 & $-15.284,9.172$ & 0.622 & \\
\hline $\mathrm{c}-\mathrm{HDL}(\mathrm{mmol} / \mathrm{L})$ & 0.079 & $-7.362,25.292$ & 0.280 & 0.171 & $\mathrm{c}-\mathrm{HDL}(\mathrm{mmol} / \mathrm{L})$ & 0.010 & $-13.590,15.323$ & 0.906 & \\
\hline $\mathrm{c}-\mathrm{LDL}(\mathrm{mmol} / \mathrm{L})$ & -0.007 & $-6.617,5.911$ & 0.912 & & $\mathrm{c}-\mathrm{LDL}(\mathrm{mmol} / \mathrm{L})$ & -0.114 & $-8.973,1.195$ & 0.133 & 0.120 \\
\hline $\mathrm{TG}(\mathrm{mmol} / \mathrm{L})$ & -0.081 & $-13.394,3.268$ & 0.232 & & $\mathrm{TG}(\mathrm{mmol} / \mathrm{L})$ & -0.088 & $-10.637,3.121$ & 0.282 & \\
\hline Glucose (mmol/L) & 0.017 & $-2.624,3.347$ & 0.812 & & Glucose (mmol/L) & 0.076 & $-1.251,3.478$ & 0.354 & \\
\hline \multirow[t]{3}{*}{ Hypertension } & 0.014 & $-15.052,18.421$ & 0.843 & & Seasonality & -0.130 & $-17.153,0.731$ & 0.072 & \\
\hline & & & & & Hypertension & -0.030 & $-15.748,10.713$ & 0.708 & \\
\hline & & & & & Leucocyte count (x10E9/L) & 0.109 & $-0.652-3.745$ & 0.167 & \\
\hline
\end{tabular}

* $\mathrm{P}$ value by multiple regression analysis.

Abbreviations: PTH Parathormone; BMI: Body Mass Index; 25(OH)D: 25.Hydroxy-cholecalcipherol CRP: C Reactive Protein.

Model 1. Potential predictors are age, gender, BML, C-HDL, C-LDL, TG, glucose, season and presence of hypertension.

Model 2. Potential predictors are age, gender, BMI, C-HDL, C-LDL, TG, glucose, season, presence of hypertension, CRP and leucocyte count. 
Table 5 Odds for prevalent metabolic syndrome, metabolic syndrome components and atherogenic dyslipidemia according to levels of PTH and 25(OH)D

\begin{tabular}{|c|c|c|c|c|c|c|c|c|}
\hline \multirow{2}{*}{$\begin{array}{l}\text { Dependent variable } \\
\text { Explanatory variables }\end{array}$} & \multicolumn{2}{|c|}{ Model I } & \multicolumn{2}{|c|}{ Model 2} & \multicolumn{2}{|c|}{ Model 3} & \multicolumn{2}{|c|}{ Model 4} \\
\hline & OR $(95 \% \mathrm{Cl})$ & $\mathrm{P}^{*}$ & OR $(95 \% \mathrm{CI})$ & $P^{*}$ & OR $(95 \% \mathrm{Cl})$ & $\mathrm{P}^{*}$ & OR $(95 \% \mathrm{Cl})$ & $\mathrm{P}^{*}$ \\
\hline \multicolumn{9}{|l|}{ Metabolic syndrome } \\
\hline PTH & $1.13(1.01,1.26)$ & 0.027 & $1.10(0.98,1.24)$ & 0.090 & $0.93(0.81,1.07)$ & 0.309 & $0.99(0.83,1.17)$ & 0.880 \\
\hline $25(\mathrm{OH})$ & $0.99(0.98,1.00)$ & 0.004 & $0.99(0.98,1.00)$ & 0.004 & $1.00(0.99,1.01)$ & 0.825 & $1.00(0.99,1.02)$ & 0.690 \\
\hline Age & & & $1.02(1.00,1.05)$ & 0.027 & $1.04(1.01,1.07)$ & 0.005 & $1.04(1.01,1.08)$ & 0.011 \\
\hline $\mathrm{BMI}$ & & & & & $1.20(1.14,1.26)$ & $<0.001$ & $1.21(1.13,1.30)$ & $<0.001$ \\
\hline \multicolumn{9}{|c|}{ Elevated blood pressure } \\
\hline PTH & $1.40(1.17,1.68)$ & $<0.001$ & $1.26(1.03,1.54)$ & 0.027 & $1.10(0.88,1.39)$ & 0.409 & $1.15(0.88,1.51)$ & 0.297 \\
\hline 25(OH)D & $1.00(0.99,1.00)$ & 0.219 & $0.99(0.98,1.00)$ & 0.141 & $1.00(0.99,1.01)$ & 0.677 & $1.00(0.98,1.01)$ & 0.825 \\
\hline Age & & & $1.07(1.04,1.10)$ & $<0.001$ & $1.06(1.03,1.10)$ & $<0.001$ & $1.03(0.99,1.07)$ & 0.117 \\
\hline $\mathrm{BMI}$ & & & & & $1.13(1.06,1.20)$ & $<0.001$ & $1.14(1.05,1.23)$ & 0.002 \\
\hline \multicolumn{9}{|c|}{ Reduced HDL cholesterol } \\
\hline PTH & $1.13(1.01,1.27)$ & 0.031 & $1.16(1.03,1.31)$ & 0.015 & $1.05(0.92,1.19)$ & 0.446 & $1.07(0.93,1.24)$ & 0.352 \\
\hline $25(\mathrm{OH}) \mathrm{D}$ & $0.99(0.98,0.99)$ & 0.001 & $0.99(0.98,0.99)$ & 0.001 & $0.99(0.99,1.00)$ & 0.180 & $1.00(0.98,1.01)$ & 0.618 \\
\hline BMl & & & & & $1.11(1.07,1.16)$ & $<0.001$ & $1.09(1.04,1.14)$ & $<0.001$ \\
\hline \multicolumn{9}{|c|}{ Elevated fasting triglycerides } \\
\hline PTH & $0.93(0.84,1.04)$ & 0.208 & $0.92(0.82,1.03)$ & 0.136 & $0.90(0.80,1.01)$ & 0.080 & $0.86(0.74,1.00)$ & 0.051 \\
\hline $25(\mathrm{OH}) \mathrm{D}$ & $0.99(0.98,1.00)$ & 0.037 & $0.99(0.98,1.00)$ & 0.024 & $0.99(0.98,1.00)$ & 0.060 & $0.98(0.97,1.00)$ & 0.026 \\
\hline Calcium Tertile 1 & $0.50(0.26,0.97)$ & 0.045 & $0.52(0.26,1.05)$ & 0.068 & $0.53(0.27,1.08)$ & 0.079 & $0.71(0.30,1.65)$ & 0.423 \\
\hline Tertile 2 & $0.51(0.27,0.97)$ & 0.040 & $0.51(0.26,0.99)$ & 0.047 & $0.50(0.25,0.97)$ & 0.041 & $1.00(0.45,2.22)$ & 0.998 \\
\hline \multicolumn{9}{|l|}{ Diabetes } \\
\hline PTH & $1.10(0.99,1.22)$ & 0.072 & $1.02(0.91,1.15)$ & 0.733 & $0.93(0.82,1.06)$ & 0.270 & $0.94(0.82,1.01)$ & 0.420 \\
\hline $25(\mathrm{OH}) \mathrm{D}$ & $0.99(0.99,1.00)$ & 0.078 & $0.99(0.99,1.00)$ & 0.023 & $1.00(0.99,1.00)$ & 0.449 & $1.00(0.99,1.01)$ & 0.787 \\
\hline Calcium Tertile 1 & $0.35(0.19,0.66)$ & 0.001 & $0.41(0.20,0.84)$ & 0.014 & $0.45(0.22,0.93)$ & 0.032 & $0.46(0.19,1.09)$ & 0.079 \\
\hline Tertile 2 & $0.44(0.24,0.80)$ & 0.008 & $0.48(0.24,0.94)$ & 0.034 & $0.43(0.21,0.88)$ & 0.021 & $0.48(0.20,1.12)$ & 0.088 \\
\hline Age & & & $1.07(1.05,1.10)$ & $<0.001$ & $1.09(1.06,1.12)$ & $<0.001$ & $1.10(1.06,1.14)$ & $<0.001$ \\
\hline $\mathrm{BMI}$ & & & & & $1.09(1.05,1.13)$ & $<0.001$ & $1.07(1.02,1.12)$ & 0.005 \\
\hline \multicolumn{9}{|c|}{ Atherogenic dyslipidemia } \\
\hline PTH & $1.22(1.07,1.40)$ & 0.004 & $1.18(1.02,1.36)$ & 0.022 & $1.07(0.92,1.25)$ & 0.345 & $1.02(0.87,1.20)$ & 0.798 \\
\hline $25(\mathrm{OH}) \mathrm{D}$ & $0.99(0.98,0.99)$ & $<0.001$ & $0.99(0.98,0.99)$ & $<0.001$ & $0.99(0.98,1.00)$ & 0.032 & $0.99(0.98,1.01)$ & 0.407 \\
\hline Calcium Tertile 1 & $0.36(0.16,0.80)$ & 0.012 & $0.41(0.18,0.92)$ & 0.032 & $0.41(0.17,0.96)$ & 0.040 & $0.50(0.19,1.29)$ & 0.153 \\
\hline Tertile 2 & $0.41(0.19,0.87)$ & 0.021 & $0.43(0.19,0.93)$ & 0.033 & $0.34(0.14,0.80)$ & 0.014 & $0.46(0.18,1.17)$ & 0.102 \\
\hline BMI & & & & & $1.09(1.05,1.14)$ & $<0.001$ & $1.11(1.05,1.17)$ & $<0.001$ \\
\hline
\end{tabular}

*P value by multiple logistic regression analysis.

PTH: Parathormone; 25(OH)D: 25.Hydroxy-cholecalcipherol; BMI: Body mass index.

Model 1. Explanatory variables in the multiple logistic regression model were PTH, 25(OH)D and tertiles of calcium.

Model 2. Explanatory variables as in model 1 plus age, gender, season and current smoking.

Model 3. Explanatory variables as in model 2 plus BMI.

Model 4. Explanatory variables as in model 3 plus uCRP and leukocyte count.

MetS even after adjustment for several confounding factors.

Higher 25(OH)D levels were associated with significantly lower odds of having atherogenic dyslipidemia (models 1, 2 and 3). This association also disappeared when the model was additionally adjusted for uCRP concentrations and leukocyte count.

\section{Discussion}

The most important finding of the present study is that both $25(\mathrm{OH}) \mathrm{D}$ and PTH were strongly associated with adiposity but not with MetS or most of its components. Several other studies have suggested that low 25(OH)D status is associated with the development of the MetS and its individual components. However, our data do 
not support an independent contribution of 25(OH)D or PTH to the pathogenesis of the MetS in a population with a wide range of adiposity. Our data are also consistent with previous reports on the high prevalence of alterations in calcium metabolism $[5,24]$ in obese subjects.

Traditionally, vitamin D has been the key regulator of serum calcium metabolism either directly or indirectly through PTH. However, vitamin D receptors are found in a wide variety of tissue, including gut, adipose tissue, cardiac and skeletal muscles, and $\beta$-cells [25]. Therefore, it is not surprising that numerous studies have investigated the potential key role of vitamin D in the pathogenesis of MetS and its individual components. As stated in the introduction section, some but not all epidemiologic studies conducted in general populations have demonstrated a relationship between low plasma $25(\mathrm{OH}) \mathrm{D}$ concentrations and the presence of MetS or its individual components [15-17]. Although these epidemiological observations are supported by mechanistic studies, experimental data are limited, especially in obese populations.

In this regard, a recent cross-sectional study conducted on 380 individuals, more than $80 \%$ of whom were overweight or obese, showed a strong association between plasma 25(OH)D concentrations and BMI [26]. Individuals with 25(OH)D deficiency had higher odds of MetS than those who had normal 25(OH)D status, probably because the authors failed to account for the confounding effect of BMI on the association between MetS and $25(\mathrm{OH}) \mathrm{D}$. In this study, no associations between plasma $25(\mathrm{OH}) \mathrm{D}$ concentrations and the individual components of MetS were shown [26].

Similar data were obtained by Botella-Carretero et al. [27] in a Spanish study conducted in a reduced sample of severely obese subjects. In this study, 25(OH)D deficiency was more prevalent in patients with the MetS than in those without. In contrast, Hjelmesaeth et al. [19], and Roislien et al. 2011 [28], failed to find any association between 25(OH)D and MetS, but reported a positive relationship between PTH plasma levels and MetS in individuals with morbid obesity. Neither was an association found between PTH or 25(OH)D and MetS in overweight or obese individuals from New Zealand [20] or Spanish morbidly obese patients [29].

There is also some controversy about the effect of low $25(\mathrm{OH}) \mathrm{D}$ status on the pathogenesis of obesity-related metabolic comorbidities and individual components of the MetS [30]. As far as glucose metabolism is concerned, Forouhi et al. [31] used data from the Medical Research Council Ely Prospective Study to show that 25 $(\mathrm{OH}) \mathrm{D}$ concentrations at baseline were inversely related with a 10-fold risk of hyperglycemia and insulin resistance. Recent studies also suggest that increased levels of
PTH are independently associated with insulin resistance in individuals with abdominal obesity suggesting a direct link between PTH and MetS [32].

Similar results have been found in many observational studies [33,34]. In contrast, the results of intervention studies are inconclusive, often because they involve the joint administration of vitamin $\mathrm{D}_{3}$ (cholecalciferol) and Ca. This makes it difficult to interpret the results, because it is not clear whether the response observed is due to vitamin $\mathrm{D}$ and/or Ca. In some randomized clinical trials (RCTs) in which cholecalciferol was used as a single treatment, insulin response to glucose improved $[35,36]$, but in others it did not. Similar findings were observed in relation to blood pressure and hypertension. Despite strong mechanistic evidence, the results of epidemiologic studies evaluating the association between 25 $(\mathrm{OH}) \mathrm{D}$ status and blood pressure are contradictory and inconclusive. The odds of having high blood pressure were lower in Australian individuals with higher 25(OH) $\mathrm{D}$ concentrations than in those with lower concentrations [37]. In contrast, 25(OH)D did not predict future risk of hypertension or increases in blood pressure in the Tromso Study [38]. Only one RCT using cholecalciferol has been conducted to determine the relationship between 25(OH)D and blood pressure. Scragg et al. [39] randomized 189 elderly subjects who received a single dose $(2.5 \mathrm{mg})$ of cholecalciferol or placebo during the winter. There was no effect on blood pressure. Likewise, our study, conducted in a large sample of individuals with different degrees of obesity, did not show any independent relationship between $25(\mathrm{OH}) \mathrm{D}$ concentrations and the odds of having diabetes and/or hypertension.

Vitamin D status has also been inversely related to atherogenic dyslipidemia in some studies $[13,40]$ but not others $[31,34,41]$. For example, plasma triglyceride concentrations were lower in US adult individuals in the top quartile of $25(\mathrm{OH}) \mathrm{D}$ than in those in the reference quartile [40]. A positive association has also been observed between 25(OH)D and plasma HDL-cholesterol concentrations [42]. No RCTs have been conducted to directly analyze the effect of vitamin $\mathrm{D}$ supplementation alone on lipid profile. However, a significant decrease in LDLcholesterol and a non-significant increase in HDLcholesterol concentrations have recently been observed after an 18-month period of oral vitamin D3 supplementation in Saudi T2DM individuals [43]. Treatment with cholecalciferol associated with energy restriction resulted in a more pronounced decrease in plasma triglyceride concentrations than energy restriction alone [44]. However, long term supplementation with low doses of cholecalciferol (5 to $20 \mu \mathrm{g}$ ) in healthy individuals had no effect on lipid profile, probably because the doses administered were not sufficient to achieve a clinically meaningful effect on lipids [45]. Other RCTs that administered 
vitamin D supplements and focused on insulin sensitivity/ release and blood pressure as the main variables reported no significant effects on lipids [35,36].

Our study demonstrated that there was an association between $25(\mathrm{OH})$ concentrations and the hypertriglyceridemia component of the MetS, even after adjusting for several confounders. It also showed an association between plasma $25(\mathrm{OH}) \mathrm{D}$ concentrations and atherogenic dyslipidemia after adjusting for such potential confounding variables as BMI, which suggests that $25(\mathrm{OH}) \mathrm{D}$ status may play a role in lipid profile. This association could be mediated by inflammation, because it disappeared when uCRP was introduced as a covariable in the analysis.

Our results confirm the results of other studies that show that obese individuals with higher BMI have a higher risk of vitamin D deficiency and elevated PTH serum concentrations. When $25(\mathrm{OH}) \mathrm{D}$ deficiency and insufficiency were merged, only $38 \%$ of individuals with a BMI $<30 \mathrm{~kg} / \mathrm{m}^{2}$ had $25(\mathrm{OH}) \mathrm{D}$ insufficiency or deficiency, compared to $88-95 \%$ of those with a BMI $>35 \mathrm{~kg} /$ $\mathrm{m}^{2}$. The prevalence of hyperparathyroidism increased from $12 \%$ in non-obese individuals to $47.5 \%$ in those with a BMI $>50 \mathrm{~kg} / \mathrm{m}^{2}$. Consistent with our results, several other studies have demonstrated an inverse relationship between BMI and 25(OH)D deficiency for both sexes, and for BMIs ranging from normal to severe obesity. In cases of severe obesity, the vitamin D abnormalities were usually more prominent. Prevalence of 25 $(\mathrm{OH}) \mathrm{D}$ deficiency or hyperparathyroidism has been observed to be as high as $>50 \%$ or $40 \%$, respectively, in individuals with a BMI $>50 \mathrm{~kg} / \mathrm{m}^{2}$. In overweight or obese women, an increase of $1 \mathrm{~kg} / \mathrm{m}^{2}$ in BMI has been associated with a decrease of $1.21 \mathrm{nmol} / \mathrm{L}$ in $25(\mathrm{OH}) \mathrm{D}$ levels [5]. Weight loss has been associated with an increase in peripheral 25(OH)D concentrations [24,46]. Therefore, our data confirm previous reports, and show that the presence of obesity is a strong predictor of hypovitaminosis $\mathrm{D}$ and hyperparathyroidism.

One reason for the increase in the prevalence of vitamin D deficiency with increasing adiposity may be the higher prevalence of non alcoholic steatohepatitis (NASH) in obesity and insulin resistance states. Vitamin D3 is converted into 25-OH-vitamin D in the liver, and vitamin $\mathrm{D}$ deficiency is common in patients with advanced liver disease. In our study we did not include those patients with advanced liver disease in order to discount the effect of this condition on vitamin D concentrations. In fact, only $1.6 \%$ of our population had ALAT values that were twice as high as the normal cutoff values (data not shown).

Some of the strengths of our study are the significant amount of clinical data that were collected in a large population of people with different degrees of obesity, and the adjustment for potential confounders such as age, gender, season of blood sampling, current smoking and BMI. However, our study also has its limitations. First, cross-sectional studies are inherently limited in that they cannot establish cause and effect relationships, and our results may not necessarily be valid in nonobese or non-white populations. Second, we acknowledge that major biases with retrospective cohort studies can impact the recall of former exposure to risk variables. Among the biases that can negatively impact the veracity of this type of study are selection bias and misclassification or information bias as a result of the retrospective aspect. Third, in our study we assumed that all individuals with a BMI higher than $35 \mathrm{~kg} / \mathrm{m}^{2}$ met the abdominal MetS criterion. This could lead to an overestimation of MetS prevalence in individuals between 35 and $40 \mathrm{~kg} / \mathrm{m}^{2}$. This overestimation is probably minor, as only $0.5 \%$ of the PREDIMED individuals [47] with a BMI between 30 and $35 \mathrm{~kg} / \mathrm{m}^{2}$ had a waist circumference lower than the criterion established for abdominal obesity (data not shown). However, abdominal obesity may have been underestimated specially in individuals between 25 and $30 \mathrm{~kg} / \mathrm{m}^{2}$. Finally, considering the diversity of results obtained in previous reports on the relationship between 25(OH)D and MetS, and the strong association found between obesity and MetS in this study, we cannot discount that the effect of BMI may have overwhelmed any marginal effect on MetS that could have been attributed to $25(\mathrm{OH}) \mathrm{D}$.

In conclusion, in our study BMI was the variable that was most strongly associated with plasma $25(\mathrm{OH}) \mathrm{D}$ and PTH concentrations. Low plasma 25(OH)D and high PTH concentrations were also associated with an increased risk of MetS, but these associations disappeared after adjustment for BMI. On the other hand, our data support a possible contribution of plasma 25 $(\mathrm{OH}) \mathrm{D}$ to the pathogenesis of hypertriglyceridemia, and atherogenic dyslipidemia through inflammation. New prospective studies will be needed to confirm these findings.

\begin{abstract}
Abbreviations
25(OH)D: 25-Hydroxy-cholecalcipherol; MetS: Metabolic syndrome; PTH: Parathormone; AD: Atherogenic dyslipidemia; BMI: Body mass index; HDL: High density lipoprotein; LDL: Low density lipoprotein; ALAT: Alanine transaminase; GT: Glutamyl transaminase; UCRP: Ultrasensitive $C$ reactive protein; ESR: Erythrocyte sedimentation rate.
\end{abstract}

\section{Competing interests}

Authors have no conflict of interest to declare.

\section{Authors' contributions}

$A G, M B$ and JSS conceived the study. AR, DDC, AB, FS and JSS recruited subjects and collected data. AG, MB and JSS performed data analysis and wrote the manuscript. AG, MB and JSS reviewed/edited the final version of the manuscript. All authors approved the final manuscript. 


\section{Acknowledgments}

We thank John Bates for the English editing of the manuscript.

\section{Funding}

This study was funded, in part, by the Spanish Ministry of Health (ISCIII), PI1001407, Thematic Network G03/140, RD06/0045, ClBERobn, and FEDER (Fondo Europeo de Desarrollo Regional). CIBERobn is an initiative of ISCIII, Spain.

\begin{abstract}
Author details
${ }^{1}$ Human Nutrition Unit, Faculty of Medicine and Health Sciences, IISPV, Universitat Rovira i Virgili, Reus, Spain. ${ }^{2}$ Human Nutrition and Dietetics, Hospital Universitari Sant Joan de Reus, IISPV, Universitat Rovira i Virgili, Reus, Spain. ${ }^{3} \mathrm{ClBER}$ obn Physiopathology of Obesity and Nutrition, Institute of Health Carlos III, Madrid, Spain. ${ }^{4}$ Surgery Service, Hospital Universitari Sant Joan de Reus, IISPV, Universitat Rovira i Virgili, Reus, Spain. ${ }^{5}$ Human Nutrition Unit. Faculty of Medicine and Health Sciences, Universitat Rovira i Virgili, C/ Sant Llorenç 21, Reus 43201, Spain.
\end{abstract}

Received: 5 September 2012 Accepted: 29 October 2012 Published: 11 December 2012

\section{References}

1. World Health Organization (WHO): Obesity and overweight:; http://www.who. int/mediacentre/factsheets/fs311/en/index.html.

2. Mithal A, Wahl DA, Bonjour JP, Burckhardt P, Dawson-Hughes B, Eisman JA, El-Hajj Fuleihan G, Josse RG, Lips P, Morales-Torres J, IOF Committee of Scientific Advisors (CSA) Nutrition Working Group: Global vitamin D status and determinants of hypovitaminosis D. Osteoporos Int 2009, 20(11):1807-1820.

3. Thacher TD, Clarke BL: Vitamin D insufficiency. Mayo Clin Proc 2011, 86(1):50-60.

4. Goldner WS, Stoner JA, Thompson J, Taylor K, Larson L, Erickson J, McBride C: Prevalence of vitamin D insufficiency and deficiency in morbidly obese patients: a comparison with non-obese controls. Obes Surg 2008, 18(2):145-150

5. Rodriguez-Rodriguez E, Navia B, Lopez-Sobaler AM, Ortega RM: Vitamin D in overweight/obese women and its relationship with dietetic and anthropometric variables. Obesity (Silver Spring) 2009, 17(4):778-782.

6. Wortsman J, Matsuoka LY, Chen TC, Lu Z, Holick MF: Decreased bioavailability of vitamin D in obesity. Am J Clin Nutr 2000, 72(3):690-693.

7. Drincic AT, Armas LA, Van Diest EE, Heaney RP: Volumetric Dilution, Rather Than Sequestration Best Explains the Low Vitamin D Status of Obesity. Obesity (Silver Spring) 2012, 20(7):1444-1448.

8. Kull M, Kallikorm R, Lember M: Body mass index determines sunbathing habits: implications on vitamin D levels. Intern Med J 2009, 39(4):256-258

9. Gemmel K, Santry HP, Prachand VN, Alverdy JC: Vitamin D deficiency in preoperative bariatric surgery patients. Surg Obes Relat Dis 2009, 5(1):54-59.

10. Zhao G, Ford ES, Li C: Associations of serum concentrations of 25hydroxyvitamin $D$ and parathyroid hormone with surrogate markers of insulin resistance among U.S. adults without physician-diagnosed diabetes: NHANES, 2003-2006. Diabetes Care 2010, 33(2):344-347.

11. Gagnon C, Lu ZX, Magliano DJ, Dunstan DW, Shaw JE, Zimmet PZ, Sikaris K, Grantham N, Ebeling PR, Daly RM: Serum 25-hydroxyvitamin D, calcium intake, and risk of type 2 diabetes after 5 years: results from a national, population-based prospective study (the Australian diabetes, obesity and lifestyle study). Diabetes Care 2011, 34(5):1133-1138.

12. Bhandari SK, Pashayan S, Liu IL, Rasgon SA, Kujubu DA, Tom TY, Sim J J: 25-hydroxyvitamin D levels and hypertension rates. J Clin Hypertens (Greenwich) 2011, 13(3):170-177.

13. Karhapaa P, Pihlajamaki J, Porsti I, Kastarinen M, Mustonen J, Niemela O, Kuusisto J: Diverse associations of 25 -hydroxyvitamin $D$ and 1,25-dihydroxy-vitamin D with dyslipidaemias. J Intern Med 2010, 268(6):604-610.

14. Reddy Vanga S, Good M, Howard PA, Vacek JL: Role of vitamin D in cardiovascular health. Am J Cardiol 2010, 10(6):798-805.

15. Hyppönen E, Boucher BJ, Berry DJ, Power C: 25-hydroxyvitamin D, IGF-1, and metabolic syndrome at 45 years of age: a cross-sectional study in the 1958 British Birth Cohort. Diabetes 2008, 57(2):298-305.
16. Lee DM, Rutter MK, O'Neill TW, Boonen S, Vanderschueren D, Bouillon R, Bartfai G, Casanueva FF, Finn JD, Forti G, Giwercman A, Han TS, Huhtaniem IT, Kula K, Lean ME, Pendleton N, Punab M, Silman AJ, Wu FC, European Male Ageing Study Group: Vitamin D, parathyroid hormone and the metabolic syndrome in middle-aged and older European men. Eur J Endocrinol 2009, 161(6):947-954.

17. Parker J, Hashmi O, Dutton D, Mavrodaris A, Stranges S, Kandala NB, Clarke A, Franco OH: Levels of vitamin D and cardiometabolic disorders: systematic review and meta-analysis. Maturitas 2010, 65(3):225-236.

18. Reis JP, von Muhlen D, Kritz-Silverstein D, Wingard DL, Barrett-Connor E: Vitamin D, parathyroid hormone levels, and the prevalence of metabolic syndrome in community-dwelling older adults. Diabetes Care 2007, 30(6):1549-1555.

19. Hjelmesaeth J, Hofso D, Aasheim ET, Jenssen T, Moan J, Hager H, Roislien J, Bollerslev J: Parathyroid hormone, but not vitamin D, is associated with the metabolic syndrome in morbidly obese women and men: a cross-sectional study. Cardiovasc Diabetol 2009, 8:7.

20. McGill AT, Stewart JM, Lithander FE, Strik CM, Poppitt SD: Relationships of low serum vitamin D3 with anthropometry and markers of the metabolic syndrome and diabetes in overweight and obesity. Nutr J 2008, 7:4.

21. Salas-Salvado J, Rubio MA, Barbany M, Moreno B, Grupo Colaborativo de la SEEDO: SEEDO 2007 Consensus for the evaluation of overweight and obesity and the establishment of therapeutic intervention criteria. Med Clin (Barc) 2007, 128(5):184-196. quiz 1 p following 200.

22. Grundy SM, Cleeman JI, Daniels SR, Donato KA, Eckel RH, Franklin BA, Gordon DJ, Krauss RM, Savage PJ, Smith SC Jr, Spertus JA, Costa F, American Heart Association, National Heart, Lung, and Blood Institute: Diagnosis and management of the metabolic syndrome: an American heart association/national heart, lung, and blood institute scientific statement. Circulation 2005, 112(17):2735-2752.

23. Ramel A, Jonsson PV, Bjornsson S, Thorsdottir I: Vitamin D deficiency and nutritional status in elderly hospitalized subjects in Iceland. Public Health Nutr 2009, 12(7):1001-1005.

24. Grethen E, McClintock R, Gupta CE, Jones R, Cacucci BM, Diaz D, Fulford AD, Perkins SM, Considine RV, Peacock M: Vitamin D and hyperparathyroidism in obesity. J Clin Endocrinol Metab 2011, 96(5):1320-1326.

25. Osei $\mathrm{K}: 25-\mathrm{OH}$ vitamin D: is it the universal panacea for metabolic syndrome and type 2 diabetes? J Clin Endocrinol Metab 2010, 95(9):4220-4222

26. Moy FM, Bulgiba A: High prevalence of vitamin D insufficiency and its association with obesity and metabolic syndrome among Malay adults in Kuala Lumpur, Malaysia. BMC Publ Health 2011, 11:735.

27. Botella-Carretero Jl, Alvarez-Blasco F, Villafruela JJ, Balsa JA, Vazquez C, Escobar-Morreale HF: Vitamin D deficiency is associated with the metabolic syndrome in morbid obesity. Clin Nutr 2007, 26(5):573-580

28. Roislien J, Van Calster B, Hjelmesaeth J: Parathyroid hormone is a plausible mediator for the metabolic syndrome in the morbidly obese: a cross-sectional study. Cardiovasc Diabetol 2011, 10:17.

29. Rueda S, Fernandez-Fernandez C, Romero F, Martinez De Osaba J, Vidal J: Vitamin D, PTH, and the metabolic syndrome in severely obese subjects. Obes Surg 2008, 18(2):151-154.

30. Muldowney S, Kiely M: Vitamin D and cardiometabolic health: a review of the evidence. Nutr Res Rev 2010, 1:1-20.

31. Forouhi NG, Luan J, Cooper A, Boucher BJ, Wareham NJ: Baseline serum 25-hydroxy vitamin $d$ is predictive of future glycemic status and insulin resistance: the medical research council Ely prospective study 1990-2000. Diabetes 2008, 57(10):2619-2625.

32. Soares MJ, Ping-Delfos WC, Sherriff JL, Nezhad DH, Cummings NK, Zhao Y: Vitamin $\mathrm{D}$ and parathyroid hormone in insulin resistance of abdominal obesity: cause or effect? Eur J Clin Nutr 2011, 65(12):1348-1352.

33. Pittas AG, Dawson-Hughes B, Li T, Van Dam RM, Willett WC, Manson JE, Hu FB: Vitamin D and calcium intake in relation to type 2 diabetes in women. Diabetes Care 2006, 29(3):650-656.

34. Liu E, Meigs JB, Pittas AG, McKeown NM, Economos CD, Booth SL, Jacques PF: Plasma 25-hydroxyvitamin d is associated with markers of the insulin resistant phenotype in nondiabetic adults. J Nutr 2009, 139(2):329-334

35. Nagpal J, Pande JN, Bhartia A: A double-blind, randomized, placebocontrolled trial of the short-term effect of vitamin D3 supplementation 
on insulin sensitivity in apparently healthy, middle-aged, centrally obese men. Diabet Med 2009, 26(1):19-27.

36. von Hurst PR, Stonehouse W, Coad J: Vitamin D supplementation reduces insulin resistance in South Asian women living in New Zealand who are insulin resistant and vitamin $D$ deficient - a randomised, placebo-controlled trial. Br J Nutr 2010, 103(4):549-555.

37. Pasco JA, Henry MJ, Nicholson GC, Brennan SL, Kotowicz MA: Behavioural and physical characteristics associated with vitamin $D$ status in women. Bone 2009, 44(6):1085-1091.

38. Jorde R, Figenschau Y, Emaus N, Hutchinson M, Grimnes G: Serum 25-hydroxyvitamin $D$ levels are strongly related to systolic blood pressure but do not predict future hypertension. Hypertension 2010, 55(3):792-798.

39. Scragg R, Khaw KT, Murphy S: Effect of winter oral vitamin D3 supplementation on cardiovascular risk factors in elderly adults. Eur I Clin Nutr 1995, 49(9):640-646.

40. Martins D, Wolf M, Pan D, Zadshir A, Tareen N, Thadhani R, Felsenfeld A, Levine B, Mehrotra R, Norris K: Prevalence of cardiovascular risk factors and the serum levels of 25 -hydroxyvitamin $D$ in the united states: data from the third national health and nutrition examination survey. Arch Intern Med 2007, 167(11):1159-1165.

41. Delvin EE, Lambert M, Levy E, O'Loughlin J, Mark S, Gray-Donald K, Paradis G: Vitamin D status is modestly associated with glycemia and indicators of lipid metabolism in French-Canadian children and adolescents. J Nutr 2010, 140(5):987-991.

42. Pilz S, Dobnig H, Nijpels G, Heine RJ, Stehouwer CD, Snijder MB, van Dam RM, Dekker JM: Vitamin D and mortality in older men and women. Clin Endocrinol (Oxf) 2009, 71(5):666-672.

43. Al-Daghri NM, Alkharfy KM, Al-Othman A, El-Kholie E, Moharram O, Alokail MS, Al-Saleh Y, Sabico S, Kumar S, Chrousos GP: Vitamin D supplementation as an adjuvant therapy for patients with T2DM: an 18-month prospective interventional study. Cardiovasc Diabetol 2012. 11(1):85.

44. Zittermann A, Frisch S, Berthold HK, Gotting C, Kuhn J, Kleesiek K, Stehle P, Koertke $H$, Koerfer R: Vitamin D supplementation enhances the beneficial effects of weight loss on cardiovascular disease risk markers. Am J Clin Nutr 2009, 89(5):1321-1327.

45. Andersen R, Brot C, Mejborn H, Molgaard C, Skovgaard LT, Trolle E, Ovesen L: Vitamin D supplementation does not affect serum lipids and lipoproteins in Pakistani immigrants. Eur J Clin Nutr 2009, 63(9):1150-1153.

46. Mason C, Xiao L, Imayama I, Duggan CR, Bain C, Foster-Schubert KE, Kong A Campbell KL, Wang CY, Neuhouser ML, Li L, Jeffery WR, Robien K, Alfano CM, Blackburn GL, McTiernan A: Effects of weight loss on serum vitamin D in postmenopausal women. Am J Clin Nutr 2011, 94(1):95-103.

47. Martinez-Gonzalez MA, Corella D, Salas-Salvado J, Ros E, Covas MI, Fiol M, Warnberg J, Aros F, Ruiz-Gutierrez V, Lamuela-Raventos RM, Lapetra J, Munoz MA, Martinez JA, Saez G, Serra-Majem L, Pinto X, Mitjavila MT, Tur JA, Portillo MP, Estruch R, PREDIMED Study Investigators: Cohort profile: design and methods of the PREDIMED study. Int J Epidemiol 2012, 41(2):377-385.

doi:10.1186/1475-2840-11-149

Cite this article as: Guasch et al:: Plasma vitamin D and parathormone are associated with obesity and atherogenic dyslipidemia: a crosssectional study. Cardiovascular Diabetology 2012 11:149.

\section{Submit your next manuscript to BioMed Central and take full advantage of:}

- Convenient online submission

- Thorough peer review

- No space constraints or color figure charges

- Immediate publication on acceptance

- Inclusion in PubMed, CAS, Scopus and Google Scholar

- Research which is freely available for redistribution

Submit your manuscript at www.biomedcentral.com/submit
Ciomed Central 